\title{
Avaliação Psicológica com Usuários de Substâncias Psicoativas (SPA): uma Revisão Sistemática da Literatura
}

Evaluación Psicológica con Usuarios de Sustancias Psicoactivas (SPA): una Revisión Sistemática de la Literatura

Psychological Evaluation with Users of Psychoactive Substances (SPA): a Systematic Review of Literature

Ingrid Michélle de Souza Santos

Universidade Federal de Pernambuco - Pernambuco - Brasil

Thallyane Rayssa da Silva Santiago

Universidade Estadual da Paraíba - Campina Grande - Brasil

Janmeyca Rayanne Venancio de Oliveira

Instituto de Ensino Superior Múltiplo - Campina Grande - Brasil

Edgley Duarte de Lima

Universidade Federal de Pernambuco - Recife - Brasil

Monilly Ramos Araujo Melo

Universidade Federal de Campina Grande - Paraíba - Brasil

\section{RESUMO}

A Avaliação Psicológica (AP) consiste em um amplo processo que possibilita o conhecimento dos fenômenos psicológicos e auxilia no delineamento de diagnósticos e prognósticos. A AP envolve uma multiplicidade de técnicas e questões que extrapolam os limites de uma denominação exata e homogênea. Este trabalho teve como objetivo realizar um breve apanhado histórico sobre o uso de substâncias psicoativas, buscando compreender de que modo esse uso é significado e posicionado durante a história, bem como entender como se dá o processo de Avaliação Psicológica com os usuários destas substâncias. Para tanto, foi realizado um levantamento sistemático da literatura com os seguintes descritores: "avaliação psicológica AND drogas, avaliação psicológica AND substâncias psicoativas, avaliação psicológica AND dependência química" nas principais bases de dados que indexam periódicos no Brasil. Percebeu-se a insuficiência da avaliação tanto psicológica como neuropsicológica para dependentes de substâncias psicoativas, indicando-nos a necessidade de validação de mais escalas e testes psicológicos para esse público, além da adequação de outras técnicas para sujeitos dependentes. Conclui-se que a AP permite identificar os possíveis prejuízos na capacidade cognitiva e no desenvolvimento das habilidades sociais dos indivíduos que fazem uso de 
substâncias psicoativas. Por meio dessa identificação, é possível realizar os encaminhamentos necessários e intervir adequadamente nas particularidades de cada caso.

Palavras-chave: Avaliação Psicológica. Substâncias Psicoativas. Dependência.

\title{
RESUMEN
}

La Evaluación Psicológica (AP) consiste em un amplio proceso que possibilita el conocimiento de los fenómenos psicológicos y auxilia em el delineamiento de diagnósticos y pronósticos. La AP involucra una multiplicidad de técnicas y cuestiones que extrapolan los límites de una denominación exacta y homogénea. Este trabajo tuvo como objetivo realizar un breve recuento histórico sobre el uso de sustâncias psicoactivas, buscando comprender de qué modo ese uso es significado y posicionado durante la historia, así como entender cómo se da el proceso de Evaluación Psicológica com los usuarios de estas sustancias. Para ello, se realizo um levantamiento sistemático de la literatura com los siguientes descriptores: "evaluación psicológica AND drogas, evaluación psicológica AND sustâncias psicoactivas, evaluación psicológica AND dependencia química" em lãs principales bases de datos que indexan periódicos en Brasil. Se percibió la insuficiencia de la evaluación tanto psicológica y neuropsicológica para dependientes de sustâncias psicoactivas, indicando nos la necesidad de validación de más escalas y pruebas psicológicas para ese público, además de la adecuación de otras técnicas para sujetos dependientes. Se concluye que la AP permite identificar los posibles perjuicios em la capacidad cognitiva y em el desarrollo de las habilidades sociales de los individuos que hacen uso de sustâncias psicoactivas. Por medio de esa identificación, esposible realizar los encaminamientos necesarios e intervenir adecuadamente em las particularidades de cada caso.

Palabras-clave: Evaluación Psicológica. Sustancias Psicoactivas. Dependencia.

\begin{abstract}
The Psychological Assessment (AP) consists of a broad process that makes possible the knowledge of the psychological phenomena and helps in the delineation of diagnoses and prognoses. The AP involves a multiplicity of techniques and questions that extrapolate the limits of an exact and homogeneous denomination. This work aimed to make a brief historical recount about the use of psychoactive substances, seeking to understand how this use is meaning and positioned during the story, as well as understand how the process of Psychological Evaluation occurs with users of these substances. For this, a systematic survey of the literature was carried out with the following descriptors: "psychological evaluation AND drugs, psychological evaluation AND psychoactive substances, psychological evaluation AND chemical dependence" in the main databases that index newspapers in Brazil. The inadequacy of psychological and neuropsychological evaluation for psychoactive substance dependents was perceived, indicating the need for validation of more scales and psychological tests for this public, in addition to the adequacy of other techniques for dependent subjects. It is concluded that PA allows identification of possible impairments in cognitive ability and in the development of the social skills of individuals who use psychoactive substances. Through this identification, it is possible to make the necessary routing and intervene appropriately in the particularities of each case.
\end{abstract}

Keywords: Psychological Evaluation; Psychoactive Substances; Dependency. 


\section{Introdução}

A Avaliação Psicológica (AP) destacase por ser uma das áreas mais tradicionais da Psicologia. Sua ascensão ocorreu concomitantemente ao próprio desenvolvimento da Psicologia enquanto área do conhecimento científico, proporcionando o desenvolvimento de um arsenal de técnicas e instrumentos que vislumbravam uma prática profissional pautadano constructo teóricocientífico que estava em curso.

\section{A AP segundo Alchieri e Cruz (2003),} refere-se:

ao modo de conhecer fenômenos e processos psicológicos por meio de procedimentos de diagnóstico e prognóstico e, ao mesmo tempo, aos procedimentos de exame propriamente ditos para criar as condições de aferição ou dimensionamento dos fenômenos e processos psicológicos conhecidos (Alchieri \&Cruz, 2003, p. 24)

Apesar de ser uma conceituação ampla, é importante esclarecer que a AP envolve uma multiplicidade de técnicas e questões que extrapolam os limites de uma denominação exata e homogênea. Nesse sentido, em um processo de Avaliação Psicológica, é importante considerar as questões contextuais que envolvem o sujeito nesse processo, com o objetivo de dirimir os reducionismos e os equívocos que podem surgir a partir de uma análise que não considere tais aspectos.

Em termos operacionais, Godoy $\mathrm{e}$ Noronha (2005) também afirmam que a AP consiste em um processo de coleta de dados, onde são utilizados métodos e técnicas de investigação, inclusive, os testes psicológicos, instrumentos exclusivos do profissional de Psicologia e que quando utilizados de forma adequada, nos dão importantes informações a respeito das pessoas que estão passando por umprocesso avaliativo.
Espera-se que a AP seja solicitada ao psicólogo em qualquer contexto e/ou lugar em que desenvolva sua prática, seja na clínica, na comunidade, nos espaços organizacionais, no âmbito jurídico, no espaço escolar, no contexto da saúde, enfim, nos mais diversos espaços de atuação do psicólogo.

Diante das diversas possibilidades de realização de AP, algumas instituições e seus públicos podem colocar limites e/ou impasses à sua realização como, por exemplo, o caso de instituições que atendem o público voltado para a drogadição. Impasses porque a demanda apresenta especificidades que, muitas vezes, torna o processo avaliativo mais difícil de ser conduzido. Somado a isso, apresenta-se o pouco número de escalas, inventários e testes psicológicos específicos para esse público, validados para o nosso país, o que contribui para tornar o processo mais árduo e complexo para o profissional.

Neste artigo, faremos um sucinto apanhado histórico sobre o uso de substâncias psicoativas, buscando compreender de que modo esse uso é significado e posicionado durante a história. Esse recorte histórico ajudanos a entender como as substâncias psicoativas foram assumindo tamanha centralidade nas discussões de cunho político-moral, o que acaba por influenciar diretamente no psiquismo do homem, elemento essencial para se considerar em um processo de Avaliação Psicológicacom os usuários destas substâncias.

\section{Breve Histórico sobre o uso de Substâncias Psicoativas (SPA)}

O uso de substâncias psicoativas (SPA) remonta ao início da humanidade. Se hoje o uso dessas substâncias divide opiniões e gera várias discussões acerca da sua legalização ou não, na antiguidade essas preocupações não assumiam tamanha centralidade. Muitas plantas psicoativas eram utilizadas em rituais, na fabricação de medicamentos, em momentos recreativos, dentre muitas outras atividades que perpassavam a vida das sociedades da época. O uso destas, não era marcado por tantas proibições e restrições como 
percebemos na atualidade, ideia que nos faz pensar que diversos fatores atravessam a relação do homem com estas substâncias (Araújo \&Moreira, 2006; Alves, 2009).

Essa relação do homem com as SPA será mediada em cada momento histórico, a partir das determinações sócio-culturais de cada contexto social. Tais determinações serviram para condicionar e regular o uso por parte dos membros de cada sociedade, inclusive, definindo os objetivos específicos que outorgavam legitimidade ao uso dessas substâncias (Ribeiro \&Araújo, 2006; Alves, 2009).

Segundo Araújo e Moreira (2006), o consumo de quase todas as SPA no Ocidente será proibido, sobretudo, a partir do final do século XIX e início do século XX, com a proibição doálcool nos Estados Unidos. A partir desse momento, as drogas passaram a assumir um status de marginalidade nessas sociedades (ocidentais), visto que estas são atravessadas por uma multiplicidade de discursos a favor ou não do uso de tais substâncias.

Atributos pejorativos e, portanto, negativos servirão para caracterizar as drogas durante a modernidade, como, por exemplo, caracterização dessas substâncias como demoníacas, ancorada no discurso religioso. Nesse sentido, Araújo e Moreira (2006) afirmam que a natureza farmacológica das drogas passará a incorporar elementos morais, sociais e culturais de cada sociedade, mediando, então, a relação do homem com as drogas.

Tal compreensão serve como elemento paraentendermos as drogas como algo que é construído, significado e interpretado sóciohistoricamente. Desse modo, questões sociais, culturais, políticas, religiosas, científicas e econômicas atravessam o objeto em questão, na medida em que é a partir do atravessamento desses elementos, que será possível a constituição de uma pluralidade de discursos acerca do uso de tais substâncias.
No entanto, para além de uma discussão e reflexão histórica, fundamentais para a compreensão do objeto estudado nesse artigo, cabe ressaltar os impactos negativos causados pelo uso abusivo de substâncias psicoativas. É consensual na literatura nacional e internacional, que o uso indiscriminado dessas substâncias provoca diversos atrasos e prejuízos no desenvolvimento e bem-estar do ser humano. Desse modo, podemos perceber os inúmeros danos em diversas áreas da vida dos usuários de SPA.

Diante das discussões até aqui elencadas, esta pesquisa buscou fazer um levantamento da literatura sobre a Avaliação Psicológica com usuários de substâncias psicoativas. Fez-se, então, uma revisãosistemática e crítica da literatura psicológica em português acerca do assunto, com vistas a contribuir para as altercaçõesque perpassam a questão aqui abordada, além de identificar as lacunas existentes na literatura que podem servir como objetos de futuras pesquisas.

\section{Método}

Nesta pesquisa, foi realizada uma revisão sistemática da literatura com os seguintes descritores: "avaliação psicológica AND drogas, avaliação psicológica AND substâncias psicoativas, avaliação psicológica AND dependência química" nas principais bases de dados que indexam periódicos no Brasil. São elas: LILACS, BVS, SciELO, PEPSIC, Portal periódicos CAPES e o IndexPsi.

Dentre os critérios de refinamento, foram considerados: a inclusão de textos publicados em português; e, a exclusão de textos coincidentes, e/ou que não tivessem seus conteúdos disponíveis de forma completae/ou que não fizessem referência direta ao tema.

Depois de selecionado o material que atendia aos critérios de refinamento, todos os textos foram lidos na íntegra de maneira 
crítica e reflexiva, com vistas a extrair as principais convergências, divergências e novas perspectivas em torno do assunto aqui analisado. Feito isso, a análise se deu a partir de dois tipos de abordagem aos dados: a primeira, quantitativa, teve como pretensão traçar o perfil da produção bibliográfica, considerando os seguintes dados: 1) quantidade de trabalhos publicados, 2) ano de publicação, 3) métodos de pesquisas utilizados, 4) tipos de produção (teórica $\mathrm{x}$ empírica), 5) áreas/teorias que se fundamentam as publicações, 6) região predominante dos pesquisadores e 7) perfil dos participantes dos estudos; a segunda, qualitativa, teve como objetivo pontuar as principais contribuições com relação ao tema abordado.

\section{Resultados}

\section{Levantamento quantitativo}

Em termos quantitativos, foram encontrados nove trabalhos referentes a temática, sendo que apenas setedeles estavam totalmente disponíveis para acesso. Essa informação está ilustrada abaixo na figura 1:

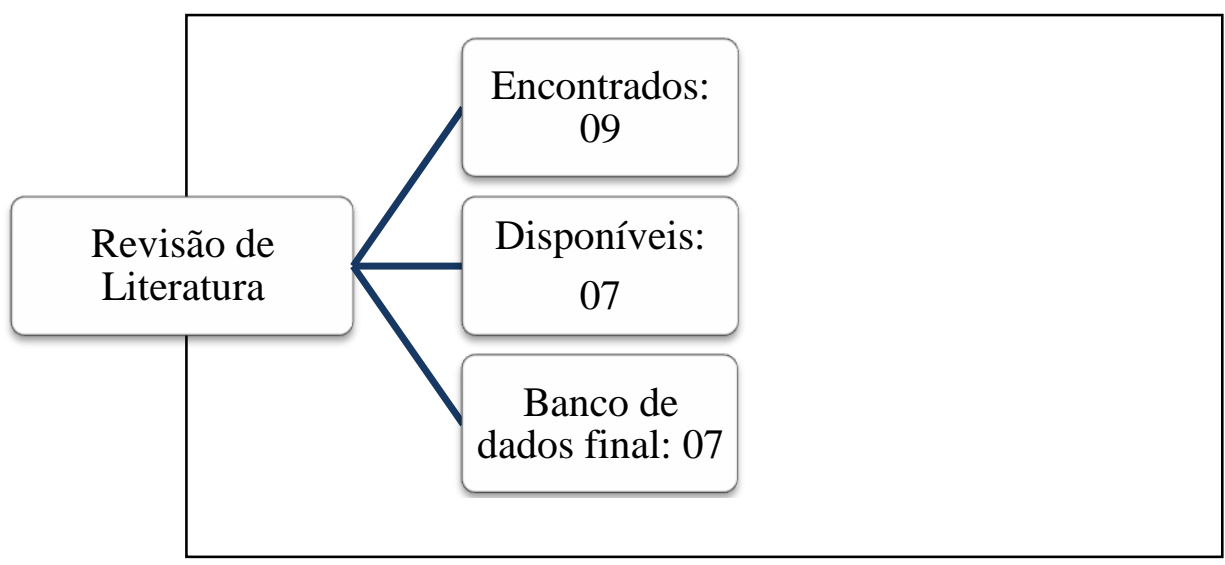

Figura 1: Detalhes da revisão de literatura

Todos os artigos do banco de dados final estão distribuídos entre os anos de 2007 a 2013, sendo um de 2007, um de 2008, um de 2011, dois de 2012 e por fim, dois de 2013. Na tabela 1, estas informações estão discorridas.

\begin{tabular}{cc}
\hline ANO DE PRODUÇÃO & QUANTIDADE \\
\hline 2007 & 01 \\
2008 & 01 \\
2011 & 01 \\
2012 & 02 \\
2013 & 02 \\
\hline TOTAL & 07 \\
\hline Tabela 1: Distribuição dos artigos por ano de produção
\end{tabular}

É relevante registrar também que dos sete trabalhos listados, seis são de natureza empírica, sendo três de natureza quantitativa e qualitativa e três são de caráter apenas quantitativo. Apenas um dos artigos é de natureza eminentemente teórica, conforme está ilustrado na tabela 2. Em relação aos métodos utilizados nos estudos empíricos, houve um prevalecimento em quase todos os artigos analisados (seis deles), do uso de escalas e questionários. 


\begin{tabular}{lc}
\hline \multicolumn{1}{c}{ TIPO DE ESTUDO } & QUANTIDADE \\
\hline Qualitativo e quantitativo & 03 \\
Quantitativo & 03 \\
Teórico & 01 \\
\hline \multicolumn{1}{c}{ TOTAL } & 07 \\
\hline
\end{tabular}

Tabela 2: Distribuição dos artigos por tipo de estudo

Sobre as áreas da Psicologia que se fundamentam os textos encontrados, se constatou o predomínio de trabalhos na área de Avaliação Psicológica (quatro deles), seguidos de trabalhos mistos (três) que não defendem uma teoria específica ou campo de estudo. A tabela 3 ilustra esses dados.

\begin{tabular}{lc}
\hline CAMPO DE ESTUDO & QUANTIDADE \\
\hline Avaliação Psicológica & 04 \\
Trabalhos mistos* & 03 \\
\hline TOTAL & 07 \\
\hline
\end{tabular}

Tabela 3: Distribuição dos artigos por campo de estudo.

* Não descreveram o campo de estudo ou teoria específica

Por fim, percebeu-se que quanto aos participantes dos estudos, constatou-se que todos os participantes eram dependentes de substâncias psicoativas e usuários de algum serviço de saúde, incluindo hospitais psiquiátricos ou fazendas de recuperação. Em todos os trabalhos percebeu-se que hegemonicamente a amostra era constituída por homens.

Com a intenção de melhor adentrar nesta temática, também fora realizado um levantamento qualitativo, compreendendo deste modo, a multidimensionalidade da Avaliação Psicológica aplicada ao contexto dos usuários de substâncias psicoativas, analisando por sua vez alguns elementos imprescindíveis de serem observados, assim como a necessidade do aprimoramento deste processo como um todo, quando aplicada a este público-alvo.

\section{Levantamento qualitativo}

A dependência química é considerada um grave problema de saúde pública, tendo em vista que ela consiste no conjunto de sintomas físicos, comportamentais e cognitivos, que indicam o uso de uma determinada substância química, causando desta maneira, grandes preocupações por parte dos profissionais de saúde em relação aos tratamentos propostos (Ciribelli, Luiz, Gorayeb, Domingos \&Filho, 2008).

O consumo destas substâncias tem sido associado ao aumento significativo nos níveis de violência e aos graves problemas de saúde. No Brasil, concomitante ao consumo, o tráfico têm chamado atenção no cenário mundial (Matumoto \&Rossini, 2013; Sousa et al., 2013).

A Classificação Internacional de Doenças (CID-10; World Health Organization, 1992) afirma que para o estabelecimento de um diagnóstico de dependência, as complicações devem ser observadas após o uso repetido de uma substância psicoativa, dispensando especial atenção para os fenômenos comportamentais, cognitivos e fisiológicos que envolvem o uso e/ou que são consequências deste.

Uma gama de estudos tem demostrado que o comportamento aditivo e, por consequente, o desenvolvimento da dependência e o processo de recaída envolvem processos cognitivos básicos, principalmente nas funções mnemônicas, executivas, atentivas e motoras (Matumoto \&Rossini, 2013). Desse modo, prejuízos neurocognitivos afetam 
diretamente a adesão ao tratamento e influenciam no processo de recaída.

As funções executivas, por exemplo, contribuem significativamente para o início das ações, pelo planejamento e previsão de recursos para solucionar problemas, adiamento de consequências e modificação de forma flexível de uma série de estratégias. No comportamento aditivo, essas funções são diretamente afetadas, contribuindo para dificuldades na compreensão e apreensão de conceitos fundamentais da terapia. Além disso, influencia no estabelecimento de um funcionamento mental desajustado no que compete ao delineamento de metas e no cumprimento de objetivos que não demandam uma recompensa imediata (Matumoto \&Rossini, 2013).

Com o intuito de avaliar os aspectos psicológicos dos usuários de substâncias psicoativas, vários estudos vêm sendo realizados, com o objetivo de identificar sinais e sintomas de ansiedade e depressão, bem como tipos de personalidade, aspectos sóciodemográficos, padrões de comportamentos, alterações dos processos cognitivos, nível motivacional, dentre outros, para assim, direcionar melhor o tratamento com dependentes de substâncias psicoativas.

Segundo Nunes e Jólluskin (2007) a avaliação do consumo de substâncias psicoativas pode impor alguns limites e entraves ao psicólogo, tornando o processo avaliativo e, consequentemente o plano terapêutico, comprometidos, caso não haja manejo por parte deste profissional.Na Avaliação Psicológica com esse público, os autores citados anteriormente indicam que $a$ priori deve-se investigar a amplitude e as circunstâncias do consumo, ou seja, o profissional deve buscar informações sobre as substâncias consumidas, o modo como são administradas, as circunstâncias e os contextos que envolvem o uso. Desse modo, interessa saber quais comportamentos contribuem para a manutenção do uso e que fatores deste uso contribuem para o condicionamento de determinados comportamentos.

Becoña e Vázquez (2001), Nunes e Jólluskin (2007) elencam alguns focos de análise pertinentes, que deverão conduzir a avaliação, a saber: as condições físicas e o estado geral do indivíduo; a coesão familiar e social; a situação econômica; a situação jurídico-legal; o estado psicológico no qual se encontra o sujeito; e o repertório comportamental. Dentre as técnicas possíveis para a avaliação dos tóxicos dependentes, estes autores destacam ainda as seguintes ferramentas: a entrevista, que tem como objetivo levantar dados mais subjetivos sobre a dinâmica psíquica do indivíduo e, nesse caso, sobre o modo pelo qual se dá o uso das substâncias psicoativas; o auto-registro, que busca identificar, tanto por parte do profissional como do indivíduo, os padrões de consumos, além das circunstâncias, pensamentos e os sentimentos do indivíduo antes e depois do uso; os instrumentos psicométricos, específicos para a avaliação de sujeitos usuários de drogas; e as provas biológicas, que permitem, através de exames, detectar o nível de consumo mais fidedignamente, visto que os usuários de drogas, frequentemente, consomem grandes quantidades de substâncias, e possuem uma falsa percepção acerca da quantidade do uso.

$\mathrm{Na}$ pesquisa realizada por Martins e Seidl (2011) com 63 pessoas que participaram de intervenções grupais do subprograma "Cessação do Tabagismo" em unidades públicas de saúde do Distrito Federal (DF), objetivando identificar características do comportamento tabagista e fatores associados à sua mudança, verificou-se que fumantes e não fumantes não se diferenciaram quanto às variáveis sociodemográficas. Dito de outra forma, a condição de estar ou não fumando não se diferenciou conforme o sexo, faixa etária, escolaridade, renda familiar ou situação conjugal na amostra analisada.

Ainda nessa pesquisa, ao avaliar a ocorrência de ansiedade e de depressão, por 
meio do Inventário Beck de Ansiedade (BAI) e do Inventário Beck de Depressão (BDI), associadas com a manutenção ou mudança do comportamento de fumar, pôde-se perceber, que, o grupo apresentou níveis mínimos e leve tanto para ansiedade $(79,4 \%)$ quanto para depressão $(84,1 \%)$. Desse modo, observou-se que há uma propensão das pessoas com níveis leves, moderados ou graves a permanecerem fumando, ao passo que as que apresentaram nível mínimo interromperam o uso de tabaco em maior frequência. Entretanto, não foram encontradas associações estatisticamente significativas dos níveis de ansiedade e de depressão e a mudança do comportamento de fumar (Martins \&Seidl, 2011).

Martins e Seidl (2011) ressaltam ainda, que o fumante deve ser apoiado e encorajado a mudar diante das dificuldades que encontra nesse processo de mudança. Assim, ao passo em que o tabagismo for reconhecido socialmente como uma doença crônica e que os aspectos negativos envolvidos no consumo de cigarro e os benefícios de sua cessação forem mais conhecidos, as concepções estigmatizantes passarão a dar lugar a uma perspectiva de cuidado e apoio.

Já a pesquisa conduzida por Matumoto e Rossini (2013) buscou analisar as alterações nos processos cognitivos, em especial a focalização atentiva e a flexibilidade mental em indivíduos diagnosticados como dependentes de drogas que passavam por um tratamento intensivo, em caráter de internaçãodia em uma universidade federal brasileira. A amostra foi composta por dois grupos de 20 pessoas, na qual o grupo 1 (G1) era caracterizado por dependentes químicos usuários do serviço e o grupo 2 (G2) formado pelo grupo controle. Em ambos, a população era hegemonicamente masculina. Os resultados demonstraram que os participantes do G1 apresentavam menor flexibilidade na resolução de problemas e funcionamento executivo rebaixado quando comparados com indivíduos do G2. Ademais, o tempo de reação do grupo de indivíduos com dependência de substâncias psicoativas foi maior que o G2, possuindo também um estado de desatenção superior ao grupo controle.

Em síntese, essa pesquisa demonstrou que os usuários de substâncias psicoativas apresentaram déficits no sistema mnemônico e lentificação no tempo de reação para uma dada resposta; fatores a serem considerados no planejamento de intervenção terapêutica para esta população, já que prejuízos neurocognitivos podem afetar a adesão ao tratamento e a sua manutenção (Matumoto \& Rossini, 2013).

Os autores atentam, ainda, para a ineficácia das técnicas de intervenção quando esses fatores são negligenciados, ou seja, as dificuldades apresentadas pelos indivíduos no que concerne a retenção de novos conhecimentos e na manutenção da atenção durante as atividades propostas. Acrescentam que a dificuldade na resolução de problemas e a diminuição da flexibilidade cognitiva impedem a adoção de novos comportamentos mais ajustados; fatos esses a serem considerados tanto no planejamento da intervenção como dito anteriormente, como nas expectativas relacionadas ao prognóstico e tratamento (Matumoto \&Rossini, 2013).

Nesse sentido, Sousa et al. (2013) sugerem que a dependência química deve ser entendida como uma doença biopsicossocial, logo, os modelos de intervenção devem incluir uma série de estratégias na abordagem da problemática. Para tanto, ressaltam que a motivação e a baixa adesão ao tratamento resultam em recaídas frequentes.

Desse modo, na pesquisa realizada pelos autores mencionados acima, utilizou-se do Modelo Transteórico de Mudança de Comportamento (Transtheoretical Model of Change), desenvolvido por James Prochaska e colaboradores em meados dos anos 70. Esse modelo afirma que até que o indivíduo chegue à prontidão para a mudança, ele transita e oscila entre quatro estágios motivacionais bem definidos, a saber: Pré-contemplação, 
Contemplação, Ação e Manutenção (Sousa et al., 2013).

No primeiro estágio, o comportamento aditivo é completamente recusado, o indivíduo não ver problema nele nem tem a intenção de mudá-lo. No segundo estágio, o indivíduo "contempla" a situação, mas se ver incapaz de modificá-la; a marca dessa fase é a ambivalência quanto à perspectiva de mudança. No estágio da Ação, as mudanças comportamentais ocorrem nitidamente, pois o indivíduo além de identificar o comportamento-problematoma atitudes e traça objetivos para transformá-lo. O último estágio, caracterizado pela estabilização é definido por uma gama de desafios a serem enfrentados para que o indivíduo não retorne a estágios anteriores; exige um esforço constante e um maior comprometimento do sujeito a ser mantido por toda a vida. No processo de avaliação é imprescindível, segundo Sousa et al. (2013), a identificação do estágio de motivação para a mudança em que o indivíduo se encontra, pois tal identificação possibilita o uso correto e eficaz de estratégias de tratamento.

Sobre aspectos da personalidade, Franco e Villemor-Amaral (2012) desenvolveram uma pesquisa com brasileiros e franceses a fim de compreender a relação espaço-temporal de cada pessoa em virtude do uso de drogas. Assim, avaliou-se a personalidade de dependentes químicos com o uso de técnicas projetivas (Zulliger e as Pirâmides Coloridas de Pfister). Constatou-se que em relação ao Zulliger, mais da metade dos dependentes químicos apresentavam dificuldades no cumprimento das regras sociais; além disso, a maioria apresentavam ainda prejuízos na capacidade de controlar e tolerar situações de estresse. Os autores justificaram essa dificuldade dos dependentes químicos em virtude da pouca presença de recursos para responder às demandas que envolvem situações estressoras ou pelo uso inadequado desses recursos. Já com relação ao Pfister, $70 \%$ dos indivíduos construíram suas pirâmides sem muita organização e uso de estratégias (construção rápida), indicando que esses sujeitos, em alguma medida e pela escolha de cores fortes, apresentavam alguma desorganização/desadaptação psíquica.

Cabe ainda destacar que quando analisadas as técnicas de maneira associada, os autores perceberam que os brasileiros, quando comparados aos franceses, mostravam-se mais angustiados, desanimados e desconfiados com relação à eficácia do tratamento. Esse resultado foi constatado, segundo os autores, pelo aumento de cores acromáticas (preto, cinza e branco) durante o processo de aplicação. Um dado importante ressaltado nessa pesquisa corresponde à relação entre o prognóstico e o processo de desintoxicação. Assim, quanto mais organizados psiquicamente estavam os sujeitos mais eles tinham um prognóstico favorável com relação à desintoxicação (Franco \&Villemor-Amaral, 2012)

Hess, Almeida e Moraes (2012), afirmam que a identificação de comorbidades psiquiátricas é importante para o prognóstico, planejamento, desenvolvimento de intervenções e tratamentos adequados.Os autores realizaram um estudo a respeito da frequência de comorbidades psiquiátricas transtornos psiquiátricos - em diferentes grupos de dependentes químicos, estando estes em abstinência e em local protegido.

Numa pesquisa de caráter exploratório quantitativo etransversal, de comparação de grupos contrastantes, Hess, Almeida e Moraes (2012) contaram com a participação de 94 homens, com idades entre 18 e 58 anos edistribuídos em quatro grupos $(\mathrm{G} 1, \mathrm{G} 2, \mathrm{G} 3 \mathrm{e}$ G4). Os grupos foram compostos por participantes não clínicos e os outros selecionados a partir do tipo de SPA consumida. O G1 era o grupo controle, o G2 composto por dependentes de álcool, o G3 por homens que associavam ao uso de álcool a maconha e o crack/cocaína e por fim, o G4, também constituído por indivíduos que associavam vários tipos de drogas, mas não apresentavam nenhuma de preferência. 
Para a avaliação, utilizou-se os seguintes instrumentos: a Ficha de Dados Sociodemográficos e o Mini International Neuropsychiatric Interview - Plus. Este último instrumento diz respeito auma entrevista diagnóstica fundamentada nos critérios do DSM-IV e na Classificação Internacional de Doenças (CID-10), cujo objetivo é avaliar os transtornos mentais ao longo da vida.

Os resultados dessa pesquisa apontaram para a existência de diferenças entre os grupos quanto à idade, a escolaridade, o estado civil e o tempo de abstinência. Os G3 e G4, compostos por pacientes que consumiam múltiplas substâncias, exibiram maior ocorrência de psicopatologias, e também, apresentaram simetria quanto ao relato de transtornos internalizantes (transtornos de humor e ansiedade) anteriores à internação atual. Não obstante, a maior frequência de indicadores de sintomas maníacos foi relatada no G3. O consumo de álcool manteve-se estável entre três grupos clínicos, diferenciando-se somente no G2, enquanto que o maior risco de suicídio (atual e passado) foi observado entre os pacientes dependentes de múltiplas substâncias psicoativas (G4) (Hess, Almeida \& Moraes, 2012).

Além disso, Hess, Almeida e Moraes (2012) averiguaram que a maioria dos participantes apresentou baixa escolaridade e, que boa parte dos pacientes usuários de drogas ilícitas eram solteiros, diferentemente do grupo, exclusivamente, alcoolista. No estudo da frequência da classe dos transtornos internalizantes, envolvendo sintomas depressivos e de ansiedade, destacou-se a alta frequência de Transtorno de Ansiedade Generalizada entre os usuários de associação de drogas ilícitas.

Outro dado relevante está relacionado ao alto índice de suicídio nos dependentes químicos, incidindo $34,5 \%$ no grupo de dependentes de múltiplas substâncias psicoativas em abstinência e $28,6 \%$ no grupo de dependentes químicos em abstinência de crack/cocaína, álcool e maconha. Ainda conforme os autores, a identificação de comorbidades psiquiátricas nesta população coopera para o bom prognóstico e para uma melhor aderência ao tratamento, bem como, para subsidiar ações de prevenção, intervenções e, também, para a elaboração de políticas públicas (Hess, Almeida \& Moraes, 2012).

Em um estudo conduzido por Ciribelli, Luiz, Gorayeb, Domingos \& Filho (2008) buscou-se verificar os benefícios de uma intervenção de apoio psicológico (baseado na Terapia Cognitivo-Comportamental) em uma sala de espera de um Ambulatório de Dependência Química. Os objetivos do estudo foi fazer um levantamento dos aspectos sociodemográficos dos pacientes, das substâncias mais utilizadas, avaliar sintomas de ansiedade e depressão e investigar a percepção dos pacientes sobre o grupo de apoio. Para isso, os autores utilizaram a Escala de Ansiedade e Depressão para Hospital Geral e o Questionário para Pacientes Atendidos no Ambulatório de Dependência Química.

Verificou-se que dos 45 pacientes que participaram da pesquisa, $84,44 \%$ eram do sexo masculino, 44,44\% estavam casados ou em união estável e $62,22 \%$ possuíam o ensino fundamental incompleto. As substâncias mais utilizadas foram cigarro $(64,44 \%)$ e álcool $(37,77 \%)$ e $71 \%$ apresentaram sintomas significativos de ansiedade e/ou depressão. Em relação a participação no grupo de apoio, os pacientes avaliaram como positiva. As razões foram as seguintes: participam porquê se sentem bem $(86,66 \%)$, para tirar dúvidas e obter informação com o psicólogo $(71,11 \%)$ e ainda para controlar o uso de drogas $(66,66 \%)$. Os pacientes relataram também que se sentiam animados, tranquilos e esclarecidos, sentindose também mais confiantes no tratamento (Ciribelli, Luiz, Gorayeb, Domingos \&Filho, 2008).

Finalmente, com base na revisão feita acima, serão pontuadas algumas questões referentes à produção acadêmica sobre a 
Avaliação Psicológica com dependentes de substâncias psicoativas, buscando identificar algumas lacunas e fazer algumas reflexões sobre o tema aqui apreciado.

\section{Discussão}

Dentre os resultados apresentados, percebeu-se a insuficiência da avaliação tanto psicológica como neuropsicológica para dependentes de substâncias psicoativas, indicando-nos a necessidade de validação de mais escalas e testes psicológicos para esse público, além da adequação de outras técnicas para sujeitos com dependência dessas substâncias.

A literatura analisada, alerta para as especificidades da avaliação com esse público, pois considera os limites e entraves no processo avaliativo com esses sujeitos, que, muitas vezes, têm suas capacidades cognitivas e habilidades sociais disfuncionais, em virtude da dependência. Nesse sentido, é preciso levar em conta as especificidades de cada substância e os seus efeitos para cada sujeito, visto que cada indivíduo reage de maneira diferente ao uso, mesmo que abusivo, dessas drogas.

Além disso, podemos perceber que em todos os estudos empíricos as variáveis sociodemográficas foram apontadas como imprescindíveis para o processo de avaliação, influenciando, sobretudo, no prognóstico e na adesão ao tratamento. Assim sendo, destacouse: a situação de risco ou vulnerabilidade que o indivíduo se encontra, o suporte familiar e social, a situação econômica e por fim a situação jurídico-legal. Sendo assim, o tratamento para a dependência química deve ser de caráter inter e multidisciplinar, tendo a participação efetiva de profissionais de diversas especialidades.

Dentre os estudos analisados, alguns estavam mais preocupados em descobrir os efeitos que essas substâncias causam no sujeito, outros muito mais em identificar as possibilidades de avaliação com técnicas e instrumentos já disponíveis ao psicólogo, o que nos aponta para a insuficiência na produção de instrumentos para esse público alvo. No entanto, não se trata de desconsiderar a relevância destes estudos, mas em pontuar a escassez de técnicas específicas para a avaliação do dependente de substâncias psicoativas.

Por fim, ressaltamos mais uma vez a pertinência de mais estudos sobre a Avaliação Psicológica com dependentes de SPA, tendo em vista que a inserção do psicólogo nas políticas públicas em saúde, sobretudo a saúde mental, ilustrada pelos serviços substitutivos (ex.: o Centro de Atenção Psicossocial álcool e drogas (CAPSad), que atende especificamente o público aqui em discussão), exige por parte deste profissional o aperfeiçoamento e a construção de novos instrumentos, não só para a Avaliação Psicológica, mas sobretudo, para auxiliar os processos terapêuticos destes sujeitos.

\section{Considerações Finais}

\section{A Avaliação Psicológica (AP)} configura-se em um processo inerente à atuação do psicólogo, podendo ser realizada nos mais diversos cenários a partir de um arsenal de instrumentos, tais como: entrevistas, observação, aplicação de testes psicométricos, projetivos e situacionais. No entanto, é válido ressaltar que tal prática é pautada em um arcabouço-teórico metodológico que auxilia ao profissional na compreensão da queixa e/ou da demanda do avaliando.

Dessa forma, cabe ressaltar que a AP enquanto método de trabalho do psicólogo possibilita inúmeros benefícios para esta população, uma vez que auxilia no prognóstico, oferece um suporte psicológico quanto à adesão do tratamento, subsidia a prevenção e intervenção, bem como, coopera com a elaboração de política pública referente aos dependentes de substâncias psicoativas.

Embora tenhamos uma série de técnicas e instrumentos psicológicos capazes de fundamentar a avaliação, percebemos que 
há um déficit de instrumentos específicos para determinados públicos, como os indivíduos que fazem uso demasiado de drogas. Dito isso, faz-se necessário validar outros testes psicológicos e escalas com o intuito de qualificar ainda mais a avaliação com esses sujeitos.

A AP permite identificar os possíveis prejuízos na capacidade cognitiva e no desenvolvimento das habilidades sociais dos indivíduos que fazem uso de SPA. Por meio dessa identificação, é possível realizar os encaminhamentos necessários, logo, se intervém adequadamente nas particularidades de cada caso.

É imprescindível ainda, que os dependentes de substâncias psicoativas, inseridos nos serviços substitutivos ou em clínicas de tratamento, sejam submetidos a uma AP, a fim de serem compreendidos em sua totalidade, para que assim possam usufruir de um projeto terapêutico individual, orientado e embasado por um viés teórico-metodológico.

\section{Referências}

Alchieri, J. C.; \&Cruz, R. M. (2003).

Avaliação Psicológica: Campo de

Conhecimento e Objetivos. In: Avaliação Psicológica: Conceitos, métodos e instrumentos. São Paulo: Casa do Psicólogo, 21-33.

Alves, V. S. (2009). Modelos de atenção à saúde de usuários de álcool e outras drogas: discursos políticos, saberes e práticas. Cad. Saúde Pública [online]. Rio de janeiro, 25(11), 2309-2319. Recuperado em 05 de abril de 2016, de http://www.scielo.br/scielo.php?script=sci arttext\&pid=S0102-311X2009001100002.

Araújo, M. R.; \&Moreira, F. G. (2006).História das drogas. In: SILVEIRA, D.X. da; MOREIRA, F. G. Panorama atual de drogas e dependências. São Paulo:

Editora Atheneu.

Becoña, E.;\&Vázquez. F.L. (2001). Heroína, Cocaína y drogas de sintesis. Madrid, Sintesis.
Ciribelli, E. B.; Luiz, A. M. A. G.; Gorayeb, R.; Domingos, N. A. M.; \& Filho, A. B. M. (2008). Intervenção em sala de espera de ambulatório de dependência química: caracterização e avaliação de efeitos. Temas em Psicologia, 16(1), 107-118. Recuperado em 05 de abril de 2016, de http://pepsic.bvsalud.org/scielo.php?script= sci_arttext\&pid=S1413389X2008000100009

Franco, R. R. C.; \&Villemor-Amaral, A. E. (2012) Validade incremental do Zulliger e do Pfister no contexto da toxicomania. Psico-USF, 17(1), p. 73- 83. Recuperado em 05 de abril de 2016, de http://www.scielo.br/scielo.php?script=sci arttext\&pid=S1413-82712012000100009

Godoy, S.L.; \&Noronha, A. P. Porto. (2005). Instrumentos psicológicos utilizados em seleção profissional. Revista do

Departamento de Psicologia - UFF, 17(1), p. 139-159. Recuperado em 05 de abril de 2016, de http://www.scielo.br/pdf/rdpsi/v17n1/v17n $\underline{1 \mathrm{a} 11}$

Hess, A. R. B.; Almeida, R. M. M.; \&Moraes, A.L. (2012). Comorbidades psiquiátricas em dependentes químicos em abstinência em ambiente protegido. Estudos de Psicologia,17(1), p. 171-178.Recuperado em 05 de abril de 2016, de http://www.scielo.br/scielo.php?script=sci arttext\&pid=S1413-294X2012000100021

Martins, K. C.; \&Seidl, E. M. F. (2011). Mudança do Comportamento de Fumar em Participantes de Grupos de Tabagismo. Psic.: Teor. e Pesq., Brasília, 27(1), 55-64. Recuperado em 05 de abril de 2016, de http://www.scielo.br/scielo.php?script=sci arttext\&pid=S0102-37722011000100008.

Matumoto, P.A.; \&Rossini, J. C. (2013). Avaliação das Funções Atentivas e Flexibilidade Mental em Dependentes Químicos. Psicologia: Reflexão e Crítica, 26(2), 339-345.Recuperado em 05 de abril de 2016, de http://www.scielo.br/scielo.php?pid=S0102 79722013000200014\&script=sci_abstract\& tlng=es.

Nunes, L.; \&Jólluskin, G. (2007) A avaliação 
como ferramenta de análise e de intervenção nas toxico dependências. Revista da Faculdade de Ciências da Saúde, Porto, 4, p. 304-313. Recuperado em 05 de abril de 2016, de http://bdigital.ufp.pt/bitstream/10284/457/1 1304-313REVISTA_FCS_04-4.pdf.

Ribeiro, M. M.; \&Araújo, M. R. (2006).

Política Mundial de drogas Ilícitas: Uma reflexão histórica. In: Silveira, D. X.; Moreira, F. G. Panorama atual de drogas e dependências. São Paulo: Editora Atheneu. Sousa, P. F.; \&Ribeiro, L. C. M.; Melo, J. R. F.; Maciel, S. C.; \& Oliveira, M. X. (2013).

Dependentes Químicos em Tratamento:

Um Estudo sobre a Motivação para

Mudança. Temas em Psicologia, 21(1), p. 259 - 268. Recuperado em 05 de abril de 2016, de http://pepsic.bvsalud.org/scielo.php?script= sci_arttext\&pid=S1413389X2013000100018.

Dados sobre as autoras:

- Ingrid Michélle de Souza Santos é graduada em Psicologia pela Universidade Federal de Campina Grande (UFCG) e Mestranda em Psicologia Cognitiva pela Universidade Federal do Pernambuco (UFPE).

- Thallyane Rayssa da Silva Santiago é graduada em Psicologia pela Universidade Federal de Campina Grande (UFCG) e aluna do curso de Pós-Graduação em Desenvolvimento Humano e Educação Escolar pela Universidade Estadual da Paraíba (UEPB).

- Janmeyca Rayanne Venancio de Oliveira é graduada em Psicologia pela Universidade Federal de Campina Grande (UFCG) e aluna do curso de Pós-Graduação em Saúde Mental e a Rede de Atenção Psicossocial pelo Instituto de Ensino Superior Múltiplo (IESM).

- Edgley Duarte de Lima é graduada em Psicologia pela Universidade Federal de Campina Grande (UFCG) e Mestranda em
Psicologia pela Universidade Federal do Pernambuco (UFPE).

- Monilly Ramos Araujo Melo é doutora em Psicologia Cognitiva pela Universidade Federal de Pernambuco (UFPE). Professora do Curso de Psicologia da Universidade Federal de Campina Grande (UFCG). Professora e Supervisora do Curso de Pós-Graduação em Neuropsicologia (UNIPÊ). Pesquisadora e Coordenadora do Laboratório de Neuropsicologia Cognitiva e Inovação Tecnológica $\quad-\quad$ LabNEUROCIT (UFCG/CNPQ). 